\title{
Simulation of fusion and quasi-fission in nuclear reactions leading to production of superheavy elements using the Constrained Molecular Dynamics model
}

\author{
J. Klimo \\ Institute of Physics, Slovak Academy of Sciences, Bratislava, Slovakia \\ M. Veselsky \\ Institute of Experimental and Applied Physics, \\ Czech Technical University, Prague, Czech Republic \\ G.A. Souliotis \\ Laboratory of Physical Chemistry, Department of Chemistry, \\ National and Kapodistrian University of Athens, Athens, Greece \\ A. Bonasera \\ Cyclotron Institute, Texas A\&M University, College Station, Texas, USA
}

\begin{abstract}
Fusion dynamics and the onset of quasi-fission in reactions, leading to production of superheavy nuclei are investigated using the constrained molecular dynamics model. Constraints on the parameters of the nuclear equation of state are derived from experimental fusion probabilities. The obtained constraint on the modulus of incompressibility of nuclear matter $K_{0}=240-260 \mathrm{MeV}$ is consistent with the results of previous study using the Boltzmann-Uehling-Uhlenbeck equation and also with constraints derived using the recent neutron star binary collision event GW170817. Unlike the modulus of incompressibility of symmetric nuclear matter, the stiffness of the density-dependence of symmetry energy influences the fusion probability only weakly.
\end{abstract}

\section{Introduction}

The microscopic description of large amplitude nuclear motion such as complete fusion, nuclear fission and quasi-fission still remains a topic of intense basic research. Understanding these processes, where participating nuclei undergo dramatic changes of their size, shape and nucleonic content, apart from a theoretical point of view, is of crucial importance for production of super-heavy nuclei and for understanding of the nucleosynthesis of heavy elements via the r-process. At present the best candidate for the event, where heavy elements are produced in the Universe, is the process of binary

Email addresses: Jozef.Klimo@savba.sk (J. Klimo), Martin.Veselsky@cvut.cz (M. Veselsky), soulioti@chem.uoa.gr (G.A. Souliotis), bonasera@tamu.edu (A. Bonasera) 
merger of neutron stars. Such an event, named GW170817, was observed recently by simultaneous detection of gravitational waves and electromagnetic signals [1], thus providing exciting opportunity for testing properties of nuclear matter, of which neutron stars consist. These properties are usually expressed in the form of the equation of state of nuclear matter, and microscopic simulations of large amplitude nuclear processes appear suitable for testing the equation of the state of nuclear matter in nuclear processes such as fusion and fission of heavy nuclei.

One of the established methods of performing microscopic simulations of large amplitude nuclear processes is the use of the microscopic model of Constrained Molecular Dynamics (CoMD) 2, 3, 4]. Simulations using this code were performed successfully for nucleus-nucleus collisions at intermediate energies, where strong nucleon exchange determines the properties of reaction products [5]. In the recent work [6] it was shown that the microscopic CoMD code is able to describe the complicated many- body dynamics of the fission process at intermediate and high energy and give a reasonable estimate of the fission time scale.

In this work we perform a systematic study of the fusion vs. quasi-fission competition in nuclear reactions, leading to production of superheavy nuclei, using the model of Constrained Molecular Dynamics. The progress in the field of production of superheavy nuclei in last two decades is summarized in the recent review article [7]. At present, the heaviest element produced, named oganesson, has atomic number $\mathrm{Z}=118$. In order to proceed further, it is necessary to understand the reaction mechanisms, competing with formation of compound nucleus, in particular the process of quasi-fission. The competition of fusion and quasi-fission was recently addressed using variety of theoretical approaches. Among others, the Dynamical Langevin approach was used in recent works [8, 9, 10]. The implementations of the quantum molecular dynamics known as ImQMD [11, 12, 13, 14] were also used recently. Furthermore, the fusion vs. quasi-fission competition was also addressed using the time-dependent Hartree-Fock theory [15, 16, 17, 18, 19, 20], and compared to measured data [21, 22]. In the recent work [23], the Boltzmann-Uehling-Uhlenbeck (BUU) equation was employed where the Pauli principle was implemented separately for neutrons and protons and the Coulomb interaction was also considered. It was possible to set a rather strict constraint on the modulus of incompressibility of the equation of state of nuclear matter $K_{0}=240-260 \mathrm{MeV}$ with density dependence of the symmetry energy within the range $\gamma=0.5-1$. In general, main effect of the nuclear equation of state in such collisions was expressed by the properties of surface, a region where density gradually drops from saturation density to zero. The fusion dynamics is governed by interplay of surface energy and Coulomb repulsion, with strong effect of symmetry energy on scission in quasifission channel. In the present work we continue this effort by employing another transport code, namely the Constrained Molecular Dynamics [2, 3, 4]. Compared to the Boltzmann-Uehling-Uhlenbeck (BUU) equation, employed in [23], the nucleons are represented in the CoMD as Gaussian wave packets and fluctuations of density are not removed by taking an average over the large set of test particles. This code was recently em- 
ployed [6] to study fission, induced by proton beam, and since fission is also governed by interplay of surface and Coulomb energy, it can be expected that the model will be suitable also for investigation of fusion vs. quasi-fission competition. It is interesting to verify the validity of the results, obtained in [23], using this version of the transport approach.

The CoMD code after necessary modification implements an effective interaction with a range of nuclear matter incompressibility of $\mathrm{K}=200-290 \mathrm{MeV}$ with several forms of the density dependence of the nucleon-nucleon symmetry potential. Moreover, via constraint imposed on the phase-space occupation for each nucleon conservation of the Pauli principle at each time step of the collision is guaranteed. A proper choice of the surface parameter of the effective interaction and the width of nucleonic wave packets has been made to describe experimentally observed fusion probabilities. The results of such simulations are described below.

\section{Constrained Molecular Dynamics}

The theoretical framework employed in this study is the microscopic model of Constrained Molecular Dynamics originally designed for reactions in the Fermi energy [2, 3, 4]. Following the general approach of Quantum Molecular Dynamics (QMD) models [24], in the CoMD code nucleons are described as localized Gaussian wave packets satisfying the uncertainty principle. The N-body phasespace distribution function is the sum of the single-particle distribution functions $f_{i}$ :

$$
f(\mathbf{r}, \mathbf{p})=\sum_{i} f_{i}(\mathbf{r}, \mathbf{p})
$$

In the present implementation of CoMD, we have taken the dispersion in momentum $\sigma_{p}$ as a parameter, in analogy to the dispersion (width of wavepacket) $\sigma_{r}$ in coordinate space. We note that $\sigma_{r}$ is a real number in the QMD approach and the distribution function $f_{i}$ reproduces the minimum uncertainty relation $\sigma_{r} \sigma_{p}=\hbar / 2$ in the one-body phase space.

With this assumption, the phase space distribution function for each nucleon takes the form:

$$
f_{i}(\mathbf{r}, \mathbf{p})=\frac{1}{\left(2 \pi \sigma_{r} \sigma_{p}\right)^{3}} \exp \left[-\frac{\left(\mathbf{r}-\left\langle\mathbf{r}_{i}\right\rangle\right)^{2}}{2 \sigma_{r}{ }^{2}}-\frac{\left(\mathbf{p}-\left\langle\mathbf{p}_{i}\right\rangle\right)^{2}}{2 \sigma_{p}^{2}}\right]
$$

We note that this distribution function can be viewed as the generalization of the classical distribution function describing pointlike particles [24]. The distribution functions $f_{i}(\mathbf{r}, \mathbf{p})$ and $f(\mathbf{r}, \mathbf{p})$ are the physical quantities of interest from which all the relevant observables are evaluated.

The equation of motion of the centroids $\left\langle\mathbf{r}_{i}\right\rangle$ and $\left\langle\mathbf{p}_{i}\right\rangle$ are deduced from the time-dependent Schrödinger equation using the time-dependent variational principle which results:

$$
\left\langle\dot{\mathbf{r}_{i}}\right\rangle=\frac{\partial H}{\partial\left\langle\mathbf{p}_{i}\right\rangle}, \quad\left\langle\dot{\mathbf{p}_{i}}\right\rangle=-\frac{\partial H}{\partial\left\langle\mathbf{r}_{i}\right\rangle}
$$


We observe that with the Gaussian description of the single-particle wave functions, the N-body timedependent Schödinger equation leads to (classical) Hamilton's equations of motion for the centroids of the nucleon wavepackets.

In the CoMD approach, the total energy $H$ for $A$ particles with mass $m$ consists of the kinetic energy and the effective interaction:

$$
H=\sum_{i} \frac{\left\langle\mathbf{p}_{i}\right\rangle^{2}}{2 m}+A \frac{3 \sigma_{p}^{2}}{2 m}+V_{e f f}
$$

The second term arises from the Gaussian width in p-space and, since it is a constant, it is ommitted in the CoMD calculations [2].

In CoMD a simplified Skyrme-like effective nucleon-nucleon interaction is adopted that leads to a potential energy $V_{\text {eff }}$ with the following terms:

$$
V_{e f f}=V^{\mathrm{vol}}+V^{(3)}+V^{\mathrm{sym}}+V^{\mathrm{surf}}+V^{\mathrm{Coul}} .
$$

where the terms of the effective interaction are, respectively, the two-body (volume) term, the threebody term, the symmetry potential, the surface term and the Coulomb term. Within CoMD model, these terms are defined as:

$$
\begin{aligned}
V^{\mathrm{vol}} & =\frac{t_{0}}{2 \rho_{0}} \sum_{i, j \neq i} \rho_{i j} \\
V^{(3)} & =\frac{t_{3}}{(\mu+1)\left(\rho_{0}\right)^{\mu}} \sum_{i, j \neq i} \rho_{i j}^{\mu}, \\
V^{\mathrm{sym}} & =\frac{a_{\mathrm{sym}}}{2 \rho_{0}^{\gamma}} \sum_{i, j \neq i}\left[2 \delta_{\tau_{i}, \tau_{j}}-1\right] \rho_{i j}^{\gamma}, \\
V^{\mathrm{surf}} & =\frac{C_{s}}{2 \rho_{0}} \sum_{i, j \neq i} \nabla_{\left\langle\mathbf{r}_{i}\right\rangle}^{2}\left(\rho_{i j}\right), \\
V^{\text {Coul }} & =\frac{1}{2} \sum_{\substack{i, j \neq i \\
(i, j \in \mathrm{prot})}} \frac{e^{2}}{\left|\left\langle\mathbf{r}_{i}\right\rangle-\left\langle\mathbf{r}_{j}\right\rangle\right|} \operatorname{erf}\left(\frac{\left|\left\langle\mathbf{r}_{i}\right\rangle-\left\langle\mathbf{r}_{j}\right\rangle\right|}{2 \sigma_{r}^{2}}\right) .
\end{aligned}
$$

In the above relations, parameters $t_{0}, t_{3}$ and exponent $\mu$ are parameters of equation of state of symmetric nuclear matter, represented by first two equations, which will be summarily characterized by the value of modulus of incompressibility $K_{0}, a_{\text {sym }}$ and exponent $\gamma$ describe the density dependence of the symmetry energy, $C_{s}$ is the coefficient describing magnitude of the surface term, $\sigma_{r}$ is the width of the nucleon wave packet in coordinate space and $\tau_{i}$ represents the z-component of the nucleon isospin degree of freedom. The superimposition integral (or interaction density) $\rho_{i j}$ is defined as:

$$
\rho_{i j} \equiv \int d^{3} r_{i} d^{3} r_{j} \rho_{i}\left(\mathbf{r}_{i}\right) \rho_{j}\left(\mathbf{r}_{j}\right) \delta\left(\mathbf{r}_{i}-\mathbf{r}_{j}\right)
$$

with the i-th nucleon density:

$$
\rho_{i} \equiv \int d^{3} p f_{i}(\mathbf{r}, \mathbf{p})
$$


The CoMD model, while not explicitly implementing antisymmetrization of the N-body wavefunction, imposes a constraint in the phase space occupation for each nucleon, effectively restoring the Pauli principle at each time step of the (classical) evolution of the system. This constraint restores, in a stochastic way, the fermionic nature of the nucleonic motion in the evolving nuclear system. The starting point of the constraint is the requirement:

$$
\begin{aligned}
& \bar{f}_{i} \leq 1 \quad(\text { for all } i) \\
& \bar{f}_{i} \equiv \sum_{j} \delta_{\tau_{i}, \tau_{j}} \delta_{s_{i}, s_{j}} \int_{h^{3}} f_{j}(\mathbf{r}, \mathbf{p}) d^{3} r d^{3} p
\end{aligned}
$$

where $s_{i}$ is the z-component of the spin of the nucleon $i$. The integral is performed in an hypercube of volume $h^{3}$ in phase space centered around the point $\left(\left\langle\mathbf{r}_{i}\right\rangle,\left\langle\mathbf{p}_{i}\right\rangle\right)$ with size $\sqrt{\frac{2 \pi \hbar}{\sigma_{r} \sigma_{p}}} \sigma_{r}$ and $\sqrt{\frac{2 \pi \hbar}{\sigma_{r} \sigma_{p}}} \sigma_{p}$ in the $r$ and $p$ spaces, respectively. Details of the computational algorithm can be found in [2].

The short range (repulsive) nucleon-nucleon interactions are described as individual nucleonnucleon collisions governed by the nucleon-nucleon scattering cross section, the available phase space and the Pauli principle, as usually implemented in transport codes (see, e.g. [25]). The handling of the Pauli-blocking in nucleon-nucleon collisions follows the aforementioned approach regarding the constraint. For each nucleon-nucleon collision, the occupation probability is evaluated after the elastic scattering and if it is less than 1 for each member of the nucleon-nucleon pair, the collision is accepted, otherwise rejected. The present CoMD version fully preserves the total angular momentum (along with linear momentum and energy), features which are critical for the accurate description of observables from heavy-ion collisions and, for the present study, the fusion/quasi-fission dynamics.

\section{Results and discussion}

In order to investigate the role of the equation of state of nuclear matter in the competition of fusion and quasi-fission in reactions leading to heavy and superheavy nuclei, we used the same representative set of reactions as in our recent work [23]. As one of the heaviest systems, where fusion is still dominant, we use the reaction ${ }^{48} \mathrm{Ca}+{ }^{208} \mathrm{~Pb}$. This reaction was measured [26, 27], and a typical dominant peak at symmetric fission was observed in the mass vs. TKE spectra of fission fragments, with TKE consistent to fusion-fission proceeding through formation of the compound nucleus ${ }^{256}$ No. Onset of quasi-fission was observed [28] in the reaction ${ }^{64} \mathrm{Ni}+{ }^{186} \mathrm{~W}$, leading to compound system ${ }^{250} \mathrm{No}$, where a prominent fusion-like peak is not observed anymore, however symmetric fission, which can be attributed to fusion-fission, is still observed relatively frequently. Quasi-fission becomes even more dominant in the reaction ${ }^{48} \mathrm{Ca}+{ }^{238} \mathrm{U}$, nominally leading to compound nucleus ${ }^{286} \mathrm{Cn}$. Nevertheless, the symmetric fission events still amount to about $10 \%$ of fission events [29]. This can be considered as upper limit for fusion probability and this value is also obtained from analysis of evaporation residue cross sections using modified HIVAP code [30, 31]. In reactions ${ }^{64} \mathrm{Ni}+{ }^{208} \mathrm{~Pb}$ [26], ${ }^{48} \mathrm{Ca}+{ }^{249} \mathrm{Cf}$ 32, 33], 

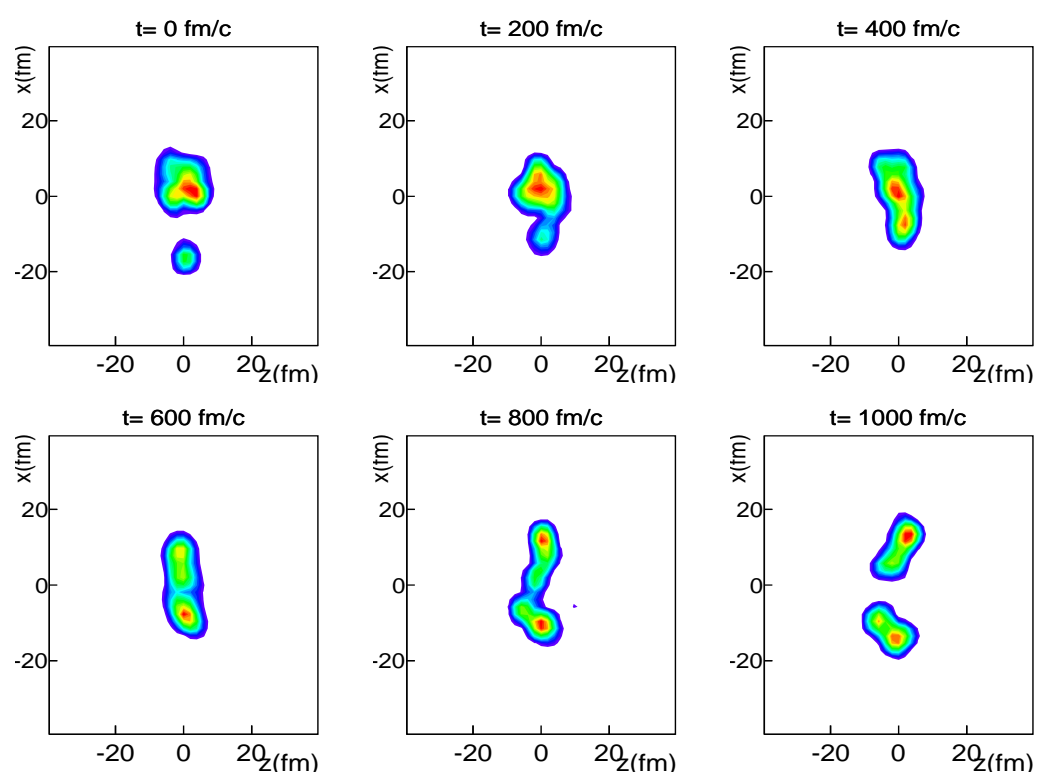

Figure 1: Typical evolution of nucleonic density for the central collision ${ }^{48} \mathrm{Ca}+{ }^{249} \mathrm{Cf}$ at beam energy $5 \mathrm{MeV} / \mathrm{nucleon}$, simulated using the equation of state with $K_{0}=245 \mathrm{MeV}$ and the density-dependence of symmetry energy with $\gamma=1$.

and ${ }^{64} \mathrm{Ni}+{ }^{238} \mathrm{U}$ [34] the quasi-fission already dominates and suppression of fusion amounts to several orders of magnitude $\left(10^{-3}-10^{-5}[30,31]\right)$. Based on the data, initial constraints on fusion were the same as in [23]. In particular, for the reaction ${ }^{48} \mathrm{Ca}+{ }^{208} \mathrm{~Pb}$ the fusion probability is close to $100 \%$, while for reactions ${ }^{64} \mathrm{Ni}+{ }^{208} \mathrm{~Pb},{ }^{48} \mathrm{Ca}+{ }^{249} \mathrm{Cf}$, and ${ }^{64} \mathrm{Ni}+{ }^{238} \mathrm{U}$ it is close to zero. This means that, considering limited number of simulated events for each case, in the former reaction one expects to observe fusion only, while in latter ones only quasi-fission. Of the two remaining reactions, the total fusion probability of $10 \%$ and the fact that fusion probability peaks at central collisions infer the constraint on fusion probability in the reaction ${ }^{48} \mathrm{Ca}+{ }^{238} \mathrm{U}$ at central events between 20 - $50 \%$ (upper limit is based on assumption that quasi-fission is dominant even in central collisions). Since comparison of shapes of experimental mass distribution in reactions ${ }^{48} \mathrm{Ca}+{ }^{238} \mathrm{U}$ and ${ }^{64} \mathrm{Ni}+{ }^{186} \mathrm{~W}$ shows that there is approximately twice higher relative abundance of fusion in reaction ${ }^{64} \mathrm{Ni}+{ }^{186} \mathrm{~W}$, we constrain the fusion probability in this reaction at central collisions between 40 - $80 \%$.

In analogy to the work [23], simulations were performed at beam energy $5 \mathrm{MeV} /$ nucleon, which is above the Coulomb barrier and in all cases corresponds to the nearest experimental point within few $\mathrm{MeV}$. The range of incompressibilities between 200 and $290 \mathrm{MeV}$ was explored. Besides the stiffness of the equation of state of symmetric nuclear matter, we implemented several assumptions on the stiffness of the density dependence of symmetry potential by varying the exponent $\gamma$ in Eq. (8) between 0.5 and 1 .

Since the angular momentum range where quasi-fission events are produced is not known precisely and also to assure that we will not observe the reactions of deep-inelastic transfer (which occur at 

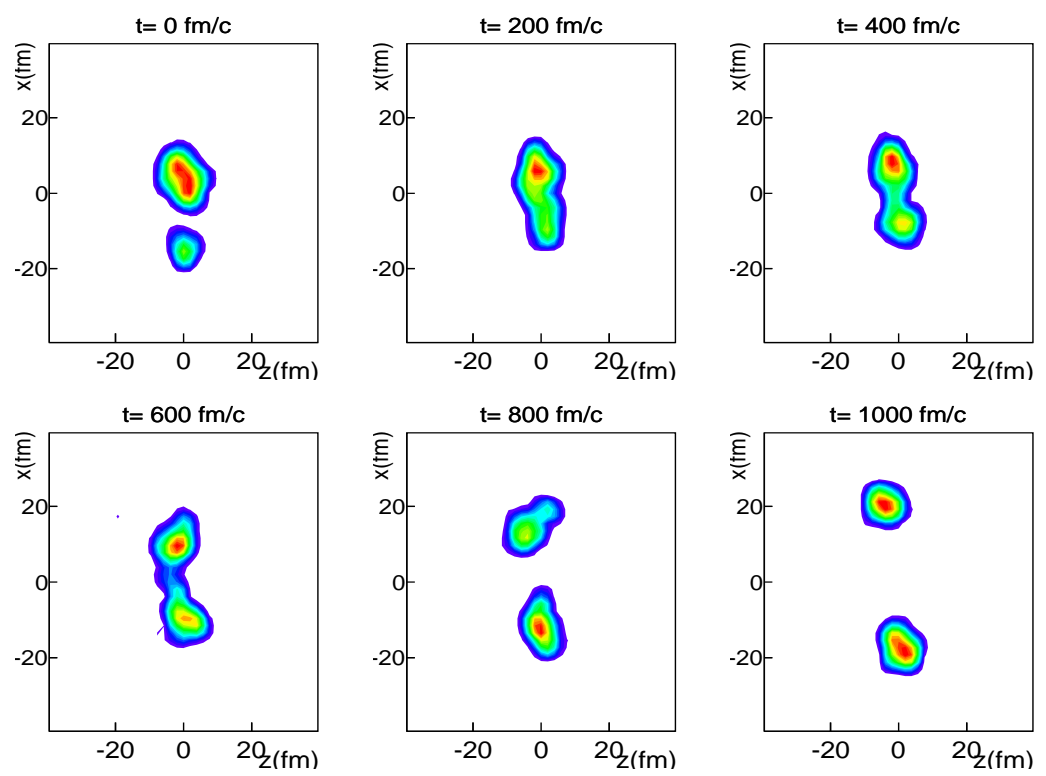

Figure 2: Typical evolution of nucleonic density for the central collision ${ }^{64} \mathrm{Ni}+{ }^{238} \mathrm{U}$ at beam energy $5 \mathrm{MeV} / \mathrm{nucleon}$, simulated using the equation of state with $K_{0}=245 \mathrm{MeV}$ and the density-dependence of symmetry energy with $\gamma=1$.

peripheral collisions) we simulated the most central events, with impact parameter set to $0.5 \mathrm{fm}$ (exactly central events practically do not occur in experiment). Simulations were performed up to the time $3000 \mathrm{fm} / \mathrm{c}$, sufficient for formation of the final configuration in all investigated cases. For each calculated reaction, the simulation was performed using 40 different sequences of the pseudorandom numbers. The simulations were performed using a computing workstation with four Xeon Phi coprocessor cards with 61 cores, allowing to perform hundreds of simulations (up to one thousand) in parallel.

Besides the parameters of the equation of state $K_{0}$ and $\gamma$, as in our previous works on fission [6], we varied also the explicit surface energy term, which is introduced in the CoMD code primarily to assure stability of the ground state configurations of the projectile and target nuclei. The corresponding parameter $\left(C_{s}\right)$ in the CoMD code varied between 0 and $-2 \mathrm{MeV} \cdot \mathrm{fm}^{2}$, the upper value meaning no additional surface energy while increasingly negative value means increasing value of the surface energy. In the present case, even such relaxation of the surface energy was not sufficient to observe quasi-fission of projectile-target combinations leading to super-heavy elements, and additional relaxation of surface energy, leading to quasi-fission, was achieved only after varying the width of the Gaussian wavepackets of the nucleons, from the standard value of $1.15 \mathrm{fm}$ down to $1 . \mathrm{fm}$. This modification of the width of Gaussian wavepackets allowed to observe quasi-fission in the heavy systems such as ${ }^{64} \mathrm{Ni}+{ }^{208} \mathrm{~Pb}$, ${ }^{48} \mathrm{Ca}+{ }^{249} \mathrm{Cf}$, and ${ }^{64} \mathrm{Ni}+{ }^{238} \mathrm{U}$. The fusion probability for these systems is by several orders of magnitude lower than sensitivity of present analysis and any set of parameters where fusion is observed must be excluded. This became a main criterion for a successful simulation and the agreement with the fusion 

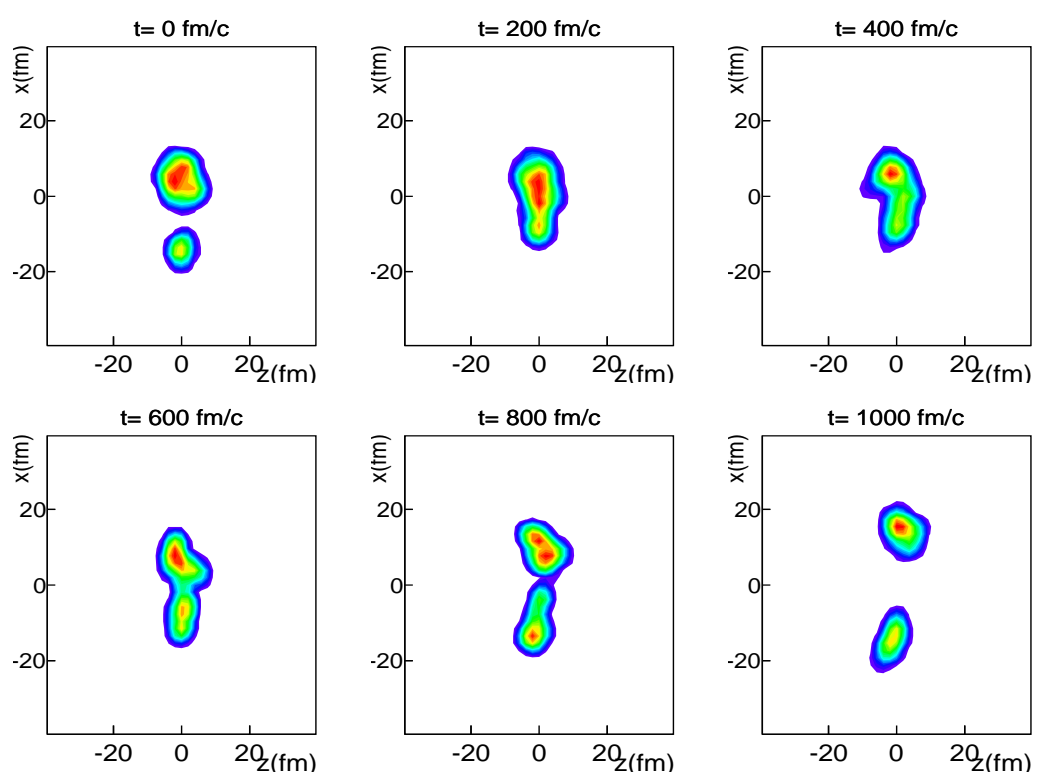

Figure 3: Typical evolution of nucleonic density for the central collision ${ }^{64} \mathrm{Ni}+{ }^{208} \mathrm{~Pb}$ at beam energy $5 \mathrm{MeV} / \mathrm{nucleon}$, simulated using the equation of state with $K_{0}=245 \mathrm{MeV}$ and the density-dependence of symmetry energy with $\gamma=1$.

in lighter systems was considered less strictly.

The analysis proceeded by performing simulations using sets of four input parameters, namely modulus of incompressibility of symmetric nuclear matter $\left(K_{0}\right)$, stiffness of the density dependence of the symmetry energy $(\gamma)$, coefficient of the explicit surface term $\left(C_{s}\right)$ and width of the Gaussian wavepacket of nucleons $\left(\sigma_{r}\right)$. The results of simulations within such four dimensional grid were compared to the experimentally observed fusion probabilities of above mentioned nuclear reactions. Compared to the work [23], where the expected values of fusion probabilities for specific reactions could be constrained within several tens of percent, in the present case the constraints had to be set somewhat more loosely. Since the focus of the work was on nuclear reactions, leading to production of superheavy elements, more emphasis was put on a correct description of behavior of the three heaviest systems, where, within sensitivity of the analysis, only quasi-fission events must be observed. However, this typically resulted in somewhat lowered probability of fusion also for lighter systems, such as ${ }^{48} \mathrm{Ca}+{ }^{176} \mathrm{Yb}$, and systems of comparable total mass, formed in reactions of beams ranging from neon to nickel. In these systems, simulations with parameter sets where no fusion was observed for heavy systems, lead to probability to observe a compound nucleus at the end of simulation of around $30 \%$. This can be possibly explained by influence of the surface energy or of the width of the nucleonic wavepackets on the exact position of the fusion barrier. This possible discrepancy in position of fusion barrier might be also a result of the missing effect of spin such as spin-orbit interaction or shell structure in CoMD simulations. In particular, deformation of target nucleus and deformed shell structure as in [19] can play a role close to the fusion barrier. Still, since the beam energy of $5 \mathrm{AMeV}$ lies above the fusion 

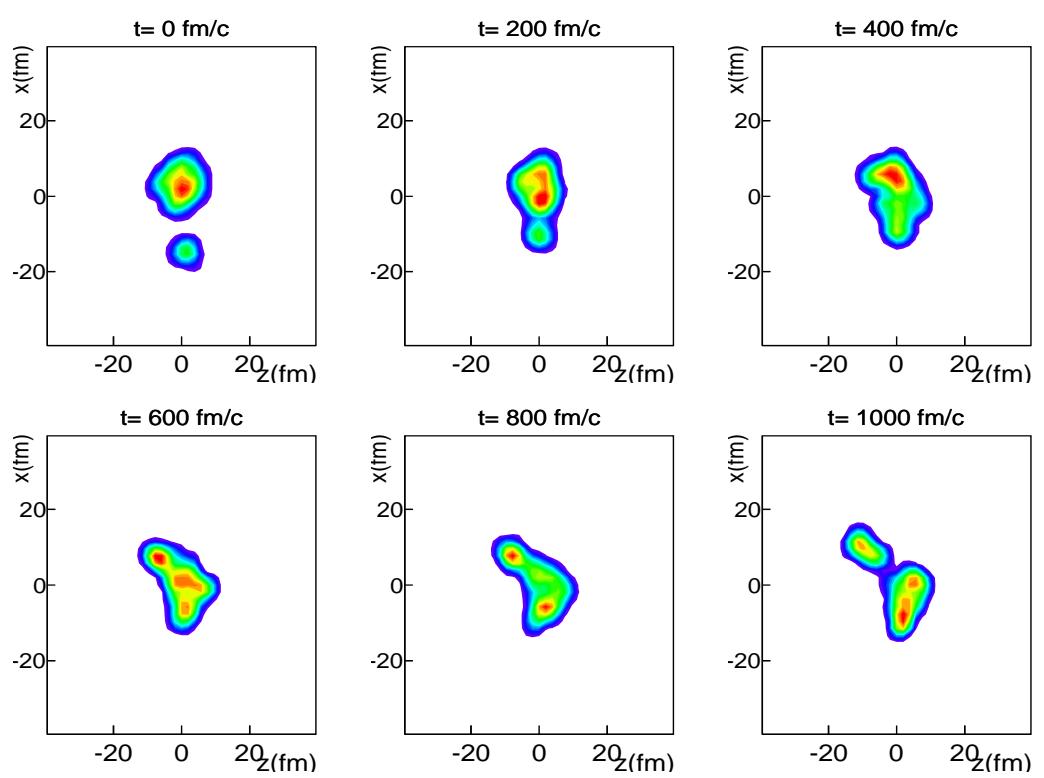

Figure 4: Typical evolution of nucleonic density for the central collision ${ }^{48} \mathrm{Ca}+{ }^{238} \mathrm{U}$ at beam energy $5 \mathrm{MeV} / \mathrm{nucleon}$, simulated using the equation of state with $K_{0}=245 \mathrm{MeV}$ and the density-dependence of symmetry energy with $\gamma=1$.

barrier, the observed level of agreement in the simulations for the whole set of reactions allows to select the most successful sets of parameters within the explored range.

The general observation from the analysis is that above some mass of the composite system $(A \approx 260)$ the balance between fusion and quasifission evolves gradually toward quasifission. No strong influence of density dependence of symmetry energy could be identified. This is again in agreement with previous fission studies using CoMD [6]. The reason for such weak dependence requires further investigations. Due to representative character of the set of selected reactions, even such loosened criteria proved to be highly selective and only two sets of parameters can be considered as most successful:

1. Modulus of incompressibility $\mathrm{K}_{0}=245 \mathrm{MeV}$, surface energy parameter $C_{s}=0 \mathrm{MeV} \cdot \mathrm{fm}^{2}$ and width of the Gaussian wavepacket $\sigma_{r}=1.085 \mathrm{fm}$

2. Modulus of incompressibility $\mathrm{K}_{0}=254 \mathrm{MeV}$, surface energy parameter $C_{s}=-1 \mathrm{MeV} \cdot \mathrm{fm}^{2}$ and width of the Gaussian wavepacket $\sigma_{r}=1 \mathrm{fm}$,

in both cases the variation of stiffness of symmetry energy $(\gamma=0.5-1)$ does not lead to observable effect on fusion probability.

In comparison with previous work using BUU model, the value of incompressibility of symmetric nuclear matter $\mathrm{K}_{0}$ between $245-254 \mathrm{MeV}$ is fully compatible, and the results of previous study appear to be confirmed. This is remarkable, since despite the fact that both BUU and CoMD are approximations to the Boltzmann equation, due to use of different implementation of smooth density (by test particle method in the former while by Gaussian wave packets in the latter case) and by use 

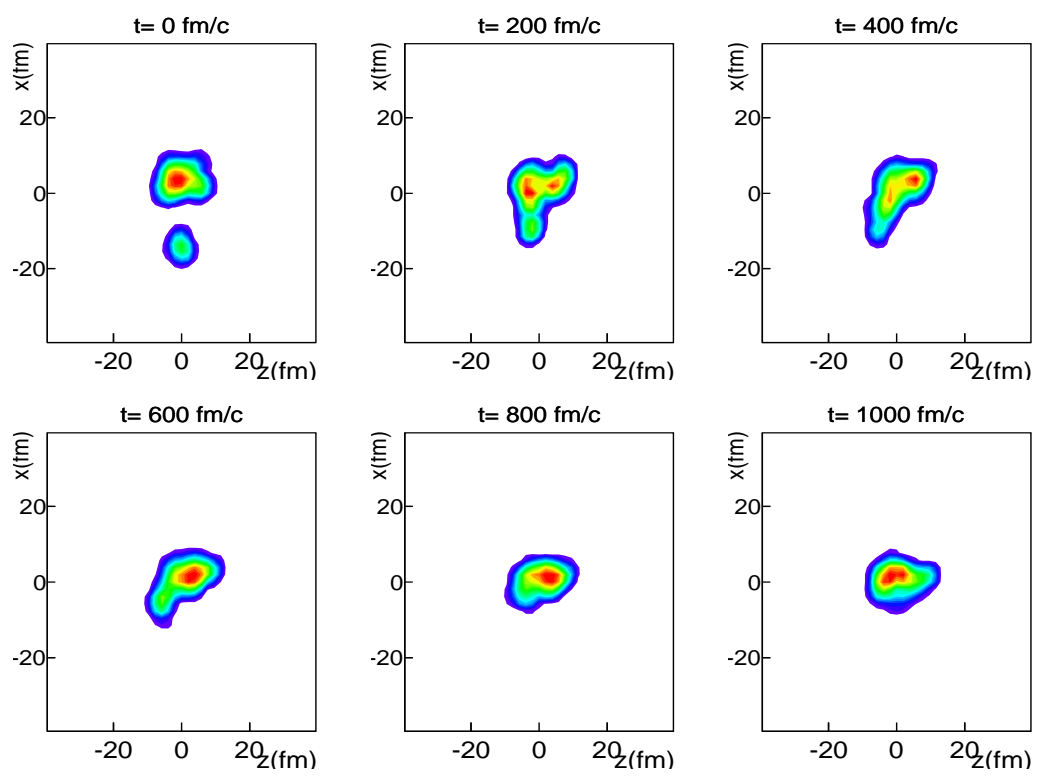

Figure 5: Typical evolution of nucleonic density for fusion of ${ }^{48} \mathrm{Ca}+{ }^{208} \mathrm{~Pb}$ at beam energy $5 \mathrm{MeV} / \mathrm{nucleon}$, simulated using the equation of state with $K_{0}=245 \mathrm{MeV}$ and the density-dependence of symmetry energy with $\gamma=1$.

of different numerical methods, such agreement is not a priori guaranteed. The two parameters $C_{s}$ and $\sigma_{r}$ appear to counterbalance each other and in order to make distinction between these two cases further investigations and comparison with other available experimental data might be necessary.

One further aspect of simulations is the difference in evaluation of the collision integral, where EoS dependent nucleon-nucleon cross sections are employed in BUU while in CoMD the collisions are driven by the experimental free nucleon-nucleon cross sections, evaluated for each case. As already mentioned in the previous work 23], specific choice of the nucleon-nucleon cross sections (EoS-dependent or free) did not lead to a dramatic effect due to strong Pauli blocking, and similar argumentation might be valid also for the case of CoMD. Still, this effect might play some role in the latter stage of quasi-fission process, where due to emergence of thermal degrees of freedom the effect of Pauli blocking will be obviously weakened.

The two successful sets of parameters were also verified in reactions of low-energy multinucleon transfer. Simulations were performed for reaction of ${ }^{136} \mathrm{Xe}+{ }^{198} \mathrm{Pt}$ at $8 \mathrm{MeV} /$ nucleon, where experimental data were obtained recently by Watanabe et al. [35]. In this reaction, the mass distributions of hot fragments, obtained using the CoMD simulations with the two above mentioned sets of parameters, were in good agreement with the mass distributions from the simulations using the model of deep-inelastic transfer (DIT) [36]. After de-excitation using the SMM code [37], the DIT simulation is capable to describe the mass distributions of cold projectile- and target-like fragments. We did not perform simulation of de-excitation of hot fragments from CoMD due to uncertainty concerning the determination of energy of the ground state of such fragments in CoMD, since the values of surface 

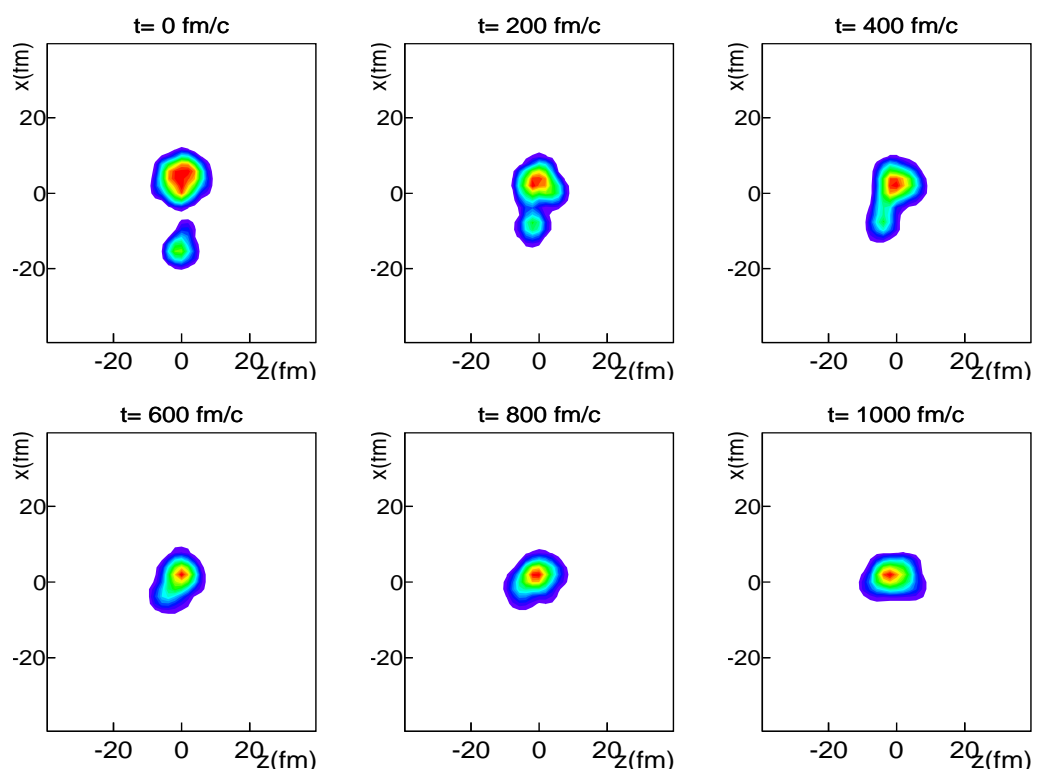

Figure 6: Typical evolution of nucleonic density for fusion of ${ }^{48} \mathrm{Ca}+{ }^{176} \mathrm{Yb}$ at beam energy $5 \mathrm{MeV} / \mathrm{nucleon}$, simulated using the equation of state with $K_{0}=245 \mathrm{MeV}$ and the density-dependence of symmetry energy with $\gamma=1$.

energy parameter $C_{s}$ and width of wavepacket $\sigma_{r}$ were not identical to the values, used to calculate properties of the ground state. However, when using the average excitation energies from DIT simulation, evaluated separately for each mass and atomic number of the fragments, we were able to reproduce the experimental data. Thus the two parameter sets appear consistent also with the experimental data from low-energy multinucleon transfer, even if more work is necessary to perform full simulations of such process using the CoMD [5].

Moreover, the recent work [38], which investigates an effect of nuclear equation of state on binary merger of neutron stars, shows that simulations of binary neutron star merger with $\mathrm{K}_{0}=245 \mathrm{MeV}$ tend to lead to formation of neutron star while softer EoS lead to formation of black hole. Subsequent astronomical observations of the recent neutron star binary merger event GW170817 report the formation of magnetar (massive neutron star) [39]. Thus the choice of nuclear EoS appears to have macroscopic consequences and the values of $\mathrm{K}_{0}=245-254 \mathrm{MeV}$ observed in the previous and present work are consistent with astronomical observations.

\section{Conclusions}

Fusion dynamics and the onset of quasi-fission in reactions, leading to production of superheavy nuclei is investigated using the constrained molecular dynamics model. Constraints on the parameters of the nuclear equation of state are derived from experimental fusion probabilities. The obtained constraint on the modulus of incompressibility of nuclear matter $K_{0}=240-260 \mathrm{MeV}$ is consistent with the results of previous study using the Boltzmann-Uehling-Uhlenbeck equation and also with 
constraints derived using the recent neutron star binary collision event GW170817. Unlike the modulus of incompressibility of symmetric nuclear matter, the stiffness of the density-dependence of symmetry energy influences the fusion probability only weakly.

\section{ACKNOWLEDGMENTS}

This work is supported by the Slovak Scientific Grant Agency under contract 2/0129/17, by the Slovak Research and Development Agency under contract APVV-15-0225 (J.K.), by the project International Mobility of Researchers in CTU CZ.02.2.69/0.0/0.0/16_027/0008465 (M.V.), by the ELKE account No 70/4/11395 of the National and Kapodistrian University of Athens (G.S.), and by the US Department of Energy-NNSA de-na0003841 (CENTAUR) (A.B.).

\section{References}

[1] B.P. Abbott, et al. (LIGO Scientific Collaboration \& Virgo Collaboration), GW170817: Observation of Gravitational Waves from a Binary Neutron Star Inspiral, Physical Review Letters. 119, (2017) 161101.

[2] M. Papa, T. Maruyama, A. Bonasera, Constrained molecular dynamics approach to fermionic systems, Phys. Rev. C 64 (2001) 024612.

[3] T. Maruyama, A. Bonasera, M. Papa, S. Chiba, Formation and decay of super-heavy systems, Eur. Phys. J. A 14 (2002) 191.

[4] M. Papa, G. Giluilani, A. Bonasera, Constrained molecular dynamics II: An N-body approach to nuclear systems, J. Comp. Phys. 208 (2005) 403.

[5] P.N. Fountas, G.A. Souliotis, M. Veselsky, A. Bonasera, Systematic study of neutron-rich rare isotope production in peripheral heavy-ion collisions below the Fermi energy, Phys. Rev. C 90 (2014) 064613.

[6] N.Vonta, G.A.Souliotis, M.Veselsky, A.Bonasera, Microscopic dynamical description of protoninduced fission with the constrained molecular dynamics model, Phys. Rev. C 92 (2015) 024616.

[7] Ch. Dullmann, R.-D. Herzberg, W. Nazarewicz, Yu.Ts. Oganessian, Special Issue on Superheavy Elements, Nucl. Phys. A 944 (2015) 1.

[8] V.I. Zagrebaev, A.V. Karpov, W. Greiner, Possibilities for synthesis of new isotopes of superheavy elements in fusion reactions, Phys. Rev. C 85 (2012) 014608. 
[9] A.V. Karpov, V.V. Saiko, Modeling near-barrier collisions of heavy ions based on a Langevin-type approach, Phys. Rev. C 96 (2017) 024618.

[10] V.V. Saiko, A.V. Karpov, Analysis of multinucleon transfer reactions with spherical and statically deformed nuclei using a Langevin-type approach, Phys. Rev. C 99 (2019) 014613.

[11] N. Wang, Z. Li, X. Wu, Improved quantum molecular dynamics model and its applications to fusion reaction near barrier, Phys. Rev. C 65 (2002) 064608.

[12] Z.Q. Feng, G.M. Jin, F.S. Zhang, Dynamical analysis on heavy-ion fusion reactions near Coulomb barrier, Nucl. Phys. A 802 (2008) 91.

[13] N. Wang, E. Zhao, S. Zhou, Theoretical study on fusion dynamics and evaporation residue cross sections for superheavy elements, Nuclear Science and Techniques 24 (2013) 050520.

[14] R.K. Choudhury, Y.K. Gupta, Revisiting the symmetric reactions for synthesis of superheavy nuclei of $Z_{i}=120$, Phys. Lett. B 731 (2014) 168.

[15] C. Golabek, C. Simenel, Collision Dynamics of Two 238U Atomic Nuclei, Phys. Rev. Lett. 103 (2009) 042701.

[16] A.S. Umar, V.E. Oberacker, J.A. Maruhn, P.-G. Reinhard, Entrance channel dynamics of hot and cold fusion reactions leading to superheavy elements, Phys. Rev. C 81 (2010) 064607.

[17] V. E. Oberacker, A. S. Umar, C. Simenel, Dissipative dynamics in quasifission, Phys. Rev. C 90 (2014) 054605.

[18] K. Sekizawa, K. Yabana, Time-dependent Hartree-Fock calculations for multinucleon transfer and quasifission processes in the $64 \mathrm{Ni}+238 \mathrm{U}$ reaction, Phys. Rev. C 93 (2016) 054616.

[19] K. Godbey, A. S. Umar, C. Simenel, Dependence of fusion on isospin dynamics, Phys. Rev. C 95 (2017) 011601.

[20] A.S. Umar, V.E. Oberacker, C. Simenel, Fusion and quasifission dynamics in the reactions 48Ca+249Bk and 50Ti+249Bk using a time-dependent Hartree-Fock approach, Phys. Rev. C 94 (2016) 024605.

[21] A. Wakhle, C. Simenel, D.J. Hinde, M. Dasgupta, M. Evers, D.H. Luong, R. du Rietz, E. Williams, Interplay between Quantum Shells and Orientation in Quasifission, Phys. Rev. Lett. 113 (2014) 182502.

[22] A. Wakhle, K. Hammerton, Z. Kohley, D. J. Morrissey, K. Stiefel, J. Yurkon, J. Walshe, K. J. Cook, M. Dasgupta, D. J. Hinde, D. J. Jeung, E. Prasad, D. C. Rafferty, C. Simenel, E. C. 
Simpson, K. Vo-Phuoc, J. King, W. Loveland, R. Yanez, Capture cross sections for the synthesis of new heavy nuclei using radioactive beams, Phys. Rev. C 97 (2018) 021602.

[23] M. Veselsky, J. Klimo, Yu-Gang Ma, G. A. Souliotis, Constraining the equation of state of nuclear matter from fusion hindrance in reactions leading to the production of superheavy elements, Phys. Rev. C 94 (2016) 064608.

[24] J. Aichelin, Quantum molecular dynamicsa dynamical microscopic n-body approach to investigate fragment formation and the nuclear equation of state in heavy ion collisions, Phys. Rep. 202 (1991) 233.

[25] A. Bonasera, F. Gulminelli, J. Molitoris, The Boltzmann equation at the borderline. A decade of Monte Carlo simulations of a quantum kinetic equation, Phys. Rep. 243 (1994) 1.

[26] R. Bock, Y.T. Chu, M. Dakowski, A. Gobbi, E. Grosse, A. Olmi, H. Sann, D. Schwalm, U. Lynen, W. Mller, S. Bjrnholm, H. Esbensen, W. Wlfli, E. Morenzoni, Dynamics of the fusion process, Nucl. Phys. A 388 (1982) 334.

[27] E.V.Prokhorova, A.A.Bogachev, M.G.Itkis, I.M.Itkis, G.N.Knyazheva, N.A.Kondratiev, E.M.Kozulin, L.Krupa, Yu.Ts.Oganessian, I.V.Pokrovsky, V.V.Pashkevich, A.Ya.Rusanov, The fusionfission and quasi-fission processes in the reaction $48 \mathrm{Ca}+208 \mathrm{~Pb}$ at energies near the Coulomb barrier, Nucl. Phys. A 802 (2008) 45.

[28] G.N. Knyazheva, M.G. Itkis, E.M. Kozulin, V.G. Lyapin, V.A. Rubchenya, W. Trzaska, S.V. Khlebnikov, The Influence of the Entrance Channel on the Formation and Decay of the Compound Nucleus 250No, PEPAN Letters 5 (2008) 40.

[29] M.G. Itkis , A.A. Bogachev, I.M. Itkis, J. Kliman, G.N. Knyazheva, N.A. Kondratiev, E.M. Kozulin, L. Krupa, Yu.Ts. Oganessian, I.V. Pokrovsky, E.V. Prokhorova, A.Ya. Rusanov, The processes of fusion-fission and quasi-fission of superheavy nuclei, Nucl. Phys. A 787 (2007) 150c.

[30] M. Veselsky, Estimation of production cross sections of superheavy elements, Acta Phys. Slovaca 49 (1999) 101.

[31] M. Veselsky, Competition of fusion and quasi-fission in the reactions leading to production of the superheavy elements, Phys. Atom. Nucl. 66 (2003) 1086; Yad. Fiz. 66 (2003) 1122.

[32] Yu.Ts.Oganessian, V.K.Utyonkov, Yu.V.Lobanov, F.Sh.Abdullin, A.N.Polyakov, R.N.Sagaidak, I.V.Shirokovsky, Yu.S.Tsyganov, A.A.Voinov, G.G.Gulbekian, S.L.Bogomolov, B.N.Gikal, A.N.Mezentsev, S.Iliev, V.G.Subbotin, A.M.Sukhov, K.Subotic, V.I.Zagrebaev, G.K.Vostokin, M.G.Itkis, K.J.Moody, J.B.Patin, D.A.Shaughnessy, M.A.Stoyer, N.J.Stoyer, P.A.Wilk, 
J.M.Kenneally, J.H.Landrum, J.F.Wild, R.W.Lougheed, Synthesis of the isotopes of elements 118 and 116 in the $249 \mathrm{Cf}$ and $245 \mathrm{Cm}+48 \mathrm{Ca}$ fusion reactions, Phys. Rev. C 74 (2006) 044602.

[33] Yu.Ts.Oganessian, F.Sh.Abdullin, C.Alexander, J.Binder, R.A.Boll, S.N.Dmitriev, J.Ezold, K.Felker, J.M.Gostic, R.K.Grzywacz, J.H.Hamilton, R.A.Henderson, M.G.Itkis, K.Miernik, D.Miller, K.J.Moody, A.N.Polyakov, A.V.Ramayya, J.B.Roberto, M.A.Ryabinin, K.P.Rykaczewski, R.N.Sagaidak, D.A.Shaughnessy, I.V.Shirokovsky, M.V.Shumeiko, M.A.Stoyer, N.J.Stoyer, V.G.Subbotin, A.M.Sukhov, Yu.S.Tsyganov, V.K.Utyonkov, A.A.Voinov, G.K.Vostokin, Production and Decay of the Heaviest Nuclei 293,294-117 and 294-118, Phys. Rev. Lett. 109 (2012) 162501.

[34] E.M. Kozulin, G.N. Knyazheva, I.M. Itkis, M.G. Itkis, A.A. Bogachev, L. Krupa, T.A. Loktev, S.V. Smirnov, V.I. Zagrebaev, J. yst, W.H. Trzaska, V.A. Rubchenya, E. Vardaci, A.M. Stefanini, M. Cinausero, L. Corradi, E. Fioretto, P. Mason, G.F. Prete, R. Silvestri, S. Beghini, G. Montagnoli, F. Scarlassara, F. Hanappe, S.V. Khlebnikov, J. Kliman, A. Brondi, A. DiNitto, R. Moro, N. Gelli, S. Szilner, Investigation of the reaction $64 \mathrm{Ni}+238 \mathrm{U}$ being an option of synthesizing element 120, Phys. Lett. B 686 (2010) 227.

[35] Y.X. Watanabe, Y. Hirayama, N. Imai, H. Ishiyama, S.C. Jeong, H. Miyatake, E. Clement, G. de France, A. Navin, M. Rejmund, C. Schmitt, G. Pollarolo, L. Corradi, E. Fioretto, D. Montanari, S.H. Choi, Y.H. Kim, J.S. Song, M. Niikura, D. Suzuki, H. Nishibata, J. Takatsu, Study of collisions of 136Xe $+198 \mathrm{Pt}$ for the KEK isotope separator, Nucl. Instr. and Meth. B 317 (2013) 752.

[36] L. Tassan-Got, C. Stefan, Deep inelastic transfers: A way to dissipate energy and angular momentum for reactions in the Fermi energy domain, Nucl. Phys. A 524 (1991) 121.

[37] J.P. Bondorf, A.S. Botvina, A.S. Iljinov, I.N. Mishustin, K. Sneppen, Statistical multifragmentation of nuclei, Phys. Rep. 257 (1995) 133.

[38] A. Perego, S. Bernuzzi, D. Radice, Thermodynamics conditions of matter in neutron star mergers, Eur. Phys. J. A 55 (2019) 124.

[39] M. van Putten, M. Della Valle, Observational evidence for Extended Emission to GW170817, Monthly Notices of the Royal Astronomical Society: Letters. 482 (2019) L46-L49; arXiv:1806.02165. 